\title{
miR-21 regulates triglyceride and cholesterol metabolism in non-alcoholic fatty liver disease by targeting HMGCR
}

\author{
CHUANZHENG SUN, FEIZHOU HUANG, XUNYANG LIU, XUEFEI XIAO, \\ MINGSHI YANG, GUI HU, HUAIZHENG LIU and LIANGKAN LIAO \\ Department of Emergency Medicine, The Third Xiang Ya Hospital \\ of Central South University, Changsha, Hunan 410013, P.R. China
}

Received September 16, 2014; Accepted January 16, 2015

DOI: $10.3892 /$ ijmm.2015.2076

\begin{abstract}
Non-alcoholic fatty liver disease (NAFLD) has emerged as a public health issue with a prevalence of $15-30 \%$ in Western populations and 6-25\% in Asian populations. Certain studies have revealed the alteration of microRNA (miRNA or miR) profiles in NAFLD and it has been suggested that miR-21 is associated with NAFLD. In the present study, we measured the serum levels of miR-21 in patients with NAFLD and also performed in vitro experiments using a cellular model of NAFLD to further investigate the effects of miR-21 on triglyceride and cholesterol metabolism. Furthermore, a novel target through which miR-21 exerts its effects on NAFLD was identified. The results revealed that the serum levels of miR-21 were lower in patients with NAFLD compared with the healthy controls. In addition, 3-hydroxy-3-methylglutaryl-co-enzyme A reductase (HMGCR) expression was increased in the serum of patients with NAFLD both at the mRNA and protein level. To mimic the NAFLD condition in vitro, HepG2 cells were treated with palmitic acid (PA) and oleic acid (OA). Consistent with the results obtained in the in vivo experiments, the expression levels of miR-21 were decreased and those of HMGCR were increased in the in vitro model of NAFLD. Luciferase reporter assay revealed that HMGCR was a direct target of miR-21 and that miR-21 exerted an effect on both HMGCR transcript degradation and protein translation. Furthermore, the results from the in vitro experiments revealed that miR-21 decreased the levels of triglycerides (TG), free cholesterol (FC) and total cholesterol (TC) in the PA/OA-treated HepG2 cells and that this effect was attenuated by HMGCR overexpression. Taken together, to the best of our knowledge, the present study is the first to report that miR-21 regulates triglyceride and cholesterol metabolism in an in vitro model of NAFLD, and that this effect is achieved by the inhibition of HMGCR expression. We specu-
\end{abstract}

Correspondence to: Professor Feizhou Huang, Department of Emergency Medicine, The Third XiangYa Hospital of Central South University, 138 Tongzipo Road, Changsha, Hunan 410013, P.R. China E-mail: feizhouh4204@163.com

Key words: miR-21, non-alcoholic fatty liver disease, HMGCR late that miR-21 may be a useful biomarker for the diagnosis and treatment of NAFLD.

\section{Introduction}

Non-alcoholic fatty liver disease (NAFLD) is a complex health condition which is characterized by the excessive accumulation of fat in the liver. It ranges from simple steatosis to non-alcoholic steatohepatitis (NASH) and, potentially, cirrhosis (1). With the increase in obesity and associated metabolic syndrome, NAFLD has emerged as a public health issue with a prevalence of $15-30 \%$ in Western populations (2-5) and 6-25\% in Asian populations (6). Accumulating evidence indicates that NAFLD is not only associated with liver-related morbidity or mortality, but also with an increased risk of coronary heart disease and other cardiovascular complications $(7,8)$. Therefore, NAFLD is a new challenge for medical researchers.

MicroRNAs (miRNAs or miRs) are non-coding RNAs $\sim 22 \mathrm{nt}$ in length, which are emerging as important regulators involved in a number of normal homeostatic processes, including the regulation of metabolic pathways, cellular stress, immune defense and inflammation (9-11). Certain studies have revealed the alteration of miRNA profiles in NAFLD (12-15). In the study by $\mathrm{Li}$ et al (12), it was demonstrated that miR-21 was downregulated in the livers of mice with diet-induced NASH. This suggests that miR-21 is associated with NAFLD. However, to the best of our knowledge, there is no report to date on the role of miR-21 in NAFLD. In the present study, we measured the serum levels of miR-21 in patients with NAFLD and also performed in vitro experiments using a cellular model of NAFLD to further investigate the effects of miR-21 on triglyceride (TG) and cholesterol metabolism. Moreover, a novel target through which miR-21 exerted its effects on NAFLD was identified.

\section{Materials and methods}

Ethics statement and patients. The study was approved by the Ethics Committee of the Third Xiangya Hospital, Central South University, Changsha, China and written informed consent was obtained from each patient prior to participation. A total of 25 patients with NAFLD and 12 healthy controls were enrolled in the present study. The blood samples were collected prior to any therapeutic procedure. Approximately 
$5 \mathrm{ml}$ of venous blood was collected from each participant. The whole blood was centrifuged at 4,000 rpm for $10 \mathrm{~min}$, then the supernatant serum was isolated and stored at $-80^{\circ} \mathrm{C}$ until analysis.

Cell culture. HepG2 cells were purchased from the American Type Culture Collection (ATCC; Manassas, VA, USA) and cultured in Dulbecco's modified Eagle's medium (DMEM; Gibco/Invitrogen Life Technologies, Carlsbad, CA, USA) supplemented with $10 \%$ fetal bovine serum (FBS; Gibco). The $\mathrm{HepG} 2$ cells were incubated at $37^{\circ} \mathrm{C}$ in $5 \% \mathrm{CO}_{2}$ humidity.

Transfection. The cells were seeded into a 6-well plate at a density of $1 \times 10^{5}$ cells/well. Transfection of cells with the miR-NC, miR-21 mimic, miR-21 inhibitor, HMGCR 3'-UTR plasmid and HMGCR overexpression plasmid was carried out using Lipofectamine 2000 (Invitrogen Life Technologies) in accordance with the manufacturer's instructions, followed by fatty acid treatment.

Fatty acid treatment. Palmitic acid (PA) and oleic acid (OA) were obtained from Sigma-Aldrich (St. Louis, MO, USA) and dissolved in isopropyl alcohol at a stock concentration of $800 \mathrm{mM}$. PA and OA were added to the culture medium at a concentration of $800 \mu \mathrm{M}$ at a 1:2 molar ratio according to the method described in the study by Cazanave et al (16). The concentration of the vehicle, isopropyl alcohol, was $1 \%$ in the final incubations.

Reverse transcription-quantitative (real-time) PCR. Total RNA was extracted from $100 \mu$ of serum and cells using a miRNeasy Mini kit (Qiagen GmbH, Hilden, Germany) in accordance with the manufacturer's instructions. Total RNA $(1 \mu \mathrm{g})$ was used as a template for reverse transcription using the First Strand cDNA Synthesis kit (Fermentas, Vilnius, Lithuania). Quantitative PCR was carried out using a SYBRGreen PCR kit (Applied Biosystems, Foster City, CA, USA) in a 7900 Sequence Detection System (Applied Biosystems). The mRNA expression level was expressed relative to the internal control using the comparative threshold cycle $(\mathrm{Ct})$ method.

Western blot analysis. Mouse monoclonal antibodies against 3-hydroxy-3-methylglutaryl-co-enzyme A reductase (HMGCR; sc-271595) and GAPDH (sc-166574) were purchased from Santa Cruz Biotechnology, Inc. (Santa Cruz, CA, USA). Proteins were extracted from the cells using a total protein extraction kit (Applygen Technologies Inc., Beijing, China) according to the manufacturer's instructions. Protein $(50 \mu \mathrm{g})$ was separated on $10 \%$ sodium dodecyl sulfate polyacrylamide gel electrophoresis gel (SDS-PAGE) and transferred onto a polyvinylidene fluoride membrane (EMD Millipore Corp., Billerica, MA, USA). The membrane was blocked with 5\% dry milk in Tris-buffered saline Tween-20 at room temperature for $1 \mathrm{~h}$. After washing 3 times with PBS, the membrane was incubated with primary antibodies (HMGCR antibody, 1:800; GAPDH antibody, $1: 2,000$ ) at $37^{\circ} \mathrm{C}$ for $2 \mathrm{~h}$, then incubated with secondary antibody (horseradish peroxidase-labeled anti-mouse antibody, 1:4,000; sc-358914) at $37^{\circ} \mathrm{C}$ for $1 \mathrm{~h}$. The signals were detected using an ECL detection kit (Pierce Biotechnology, Inc., Rockford, IL, USA).
Luciferase reporter assay. The 3'-untranslated region (UTR) segment of HMGCR and its mutant were amplified and inserted into the pGL3-control luciferase reporter vector (Promega, Madison, WI, USA). The cells were co-transfected with the miR-NC/miR-21 mimic and HMGCR 3'-UTR using Lipofectamine 2000 reagent (Invitrogen Life Technologies) according to the manufacturer's instructions. Forty-eight hours later, the cell lysates were prepared and luciferase activity was measured using a luciferase assay kit (Promega).

Measurement of TG, free cholesterol (FC) and total cholesterol (TC) levels. The levels of TG, FC and TC in the HepG2 cells were determined using commercial TG, FC and TC assay kits from Solarbio Inc. (Shanghai, China) following the manufacturer's instructions. Briefly, the cells were lysed in ice-cold cell lysis buffer and centrifuged at 1,500 $\mathrm{x} \mathrm{g}$ for $5 \mathrm{~min}$. The supernant was used for the determination of TG, FC and TC levels.

Statistical analysis. All data are presented as the means \pm standard deviation. A two-tailed Student's t-test was used to analyze the statistical difference between 2 groups with SPSS 19.0 statistical software (SPSS, Inc., Chicago, IL, USA). A value of $\mathrm{P}<0.05$ was considered to indicate a statistically significant difference.

\section{Results}

Expression of miR-21 and HMGCR in patients with NAFLD. We investigated whether miR-21 is found in serum from patients with NAFLD by RT-qPCR. As shown in Fig. 1, the serum levels of miR-21 were significantly downregulated in the patients with NAFLD compared with the healthy controls $(\mathrm{P}<0.05)$.

In addition, HMGCR mRNA and HMGCR protein levels in serum were determined by RTqPCR and western blot analysis, respectively. The mRNA level of HMGCR was significantly increased in the patients with NAFLD compared with the healthy controls $(\mathrm{P}<0.01$; Fig. $2 \mathrm{~A})$. As shown in Fig. $2 \mathrm{~B}$, the protein level of HMGCR was also higher in the patients with NAFLD than in the healthy controls $(\mathrm{P}<0.05)$.

Levels of TG, FC and TC in the in vitro model of NAFLD. The HepG2 cells were treated with PA and OA to establish the in vitro model of NAFLD. Compared with the vehicletreated cells, the level of TG in the PA/OA-treated cells was significantly increased ( $\mathrm{P}<0.01$; Fig. 3A). The levels of $\mathrm{FC}$ and TC were further examined. As shown in Fig. 3B and $C$, the levels of FC and TC were significantly increased in the PA/ OA-treated cells compared with the vehicle-treated cells.

Expression of miR-21 and HMGCR in the in vitro model of NAFLD. As demonstrated by RT-qPCR (Fig. 4), the PA/ OA-treated HepG2 cells showed a decreased expression of miR-21 $(\mathrm{P}<0.01)$. However, the expression of HMGCR was increased in the PA/OA-treated HepG2 cells at the mRNA and protein level compared with the vehicle-treated HepG2 cells (Fig. 5).

miR-21 directly targets the HMGCR 3'-UTR and regulates HMGCR expression. To investigate whether miR-21 regulates HMGCR expression, miR-21 mimic and miR-21 inhibitor 


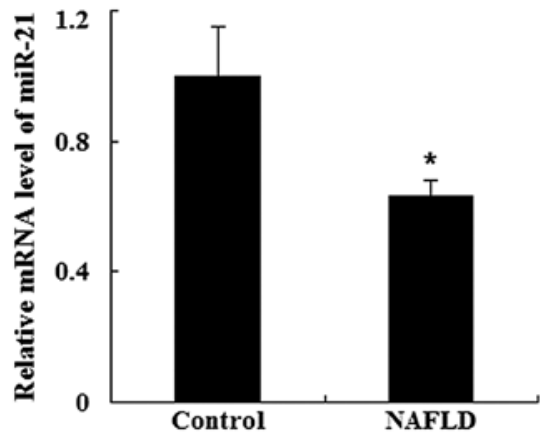

Figure 1. Expression of miR-21 in the serum of healthy controls and patients with non-alcoholic fatty liver disease (NAFLD). " $\mathrm{P}<0.05$ compared with the controls.
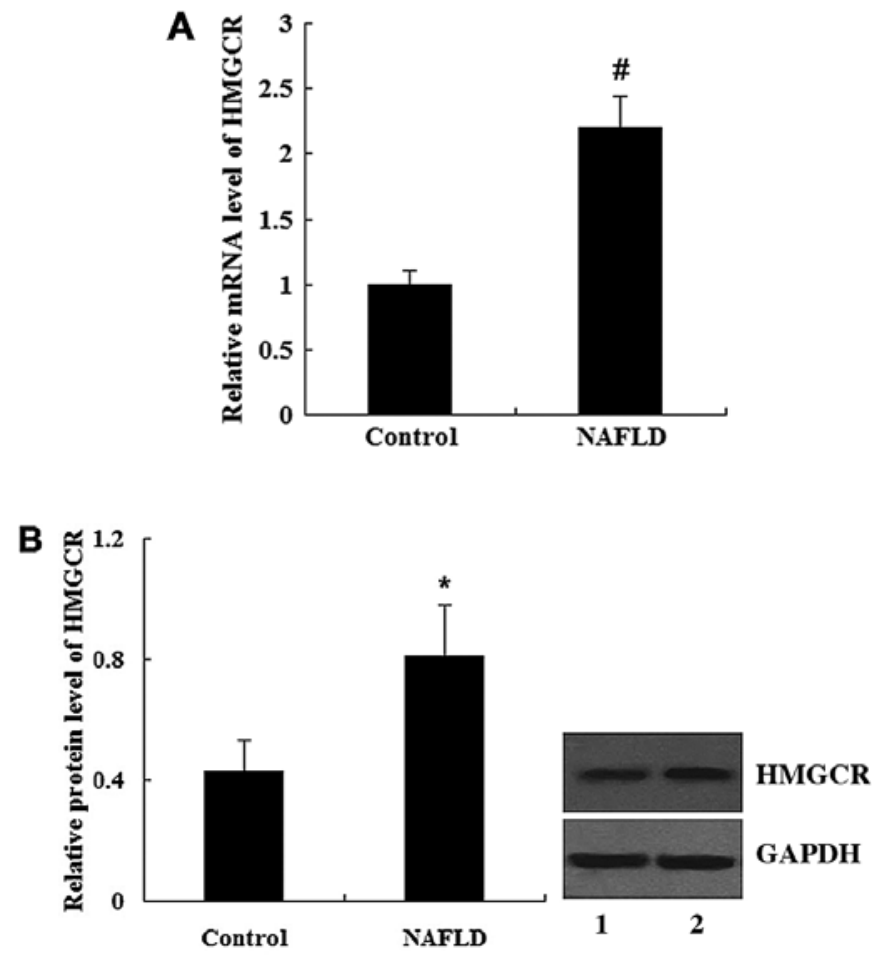

Figure 2. Expression of HMGCR in the serum of healthy controls and patients with non-alcoholic fatty liver disease (NAFLD). (A) Relative mRNA level of HMGCR. (B) Relative protein level of HMGCR. Lane 1, control group; lane 2, NAFLD group. ${ }^{*} \mathrm{P}<0.05$ and ${ }^{\#} \mathrm{P}<0.01$ compared with the controls. HMGCR, 3-hydroxy-3-methylglutaryl-co-enzyme A reductase

were transfected into the HepG2 cells. It was demonstrated that the relative mRNA level of miR-21 was significantly increased in the miR-21 mimic-transfected cells, but decreased in the miR-21 inhibitor-transfected cells compared with the miR-NC-transfected cells ( $\mathrm{P}<0.01$; Fig. 6).

To determine whether HMGCR is a direct target of miR-21, HMGCR wild-type 3'-UTR or HMGCR mutated 3'-UTR was cloned into the luciferase reporter vector. As shown in Fig. 7, the luciferase activity was significantly decreased in the cells co-transfected with the HMGCR wild-type 3'-UTR and the miR-21 mimic $(\mathrm{P}<0.05)$. However, transfection with the miR-21 mimic did not have any effect on the luciferase activity of the HMGCR mutated 3'-UTR $(\mathrm{P}>0.05)$.
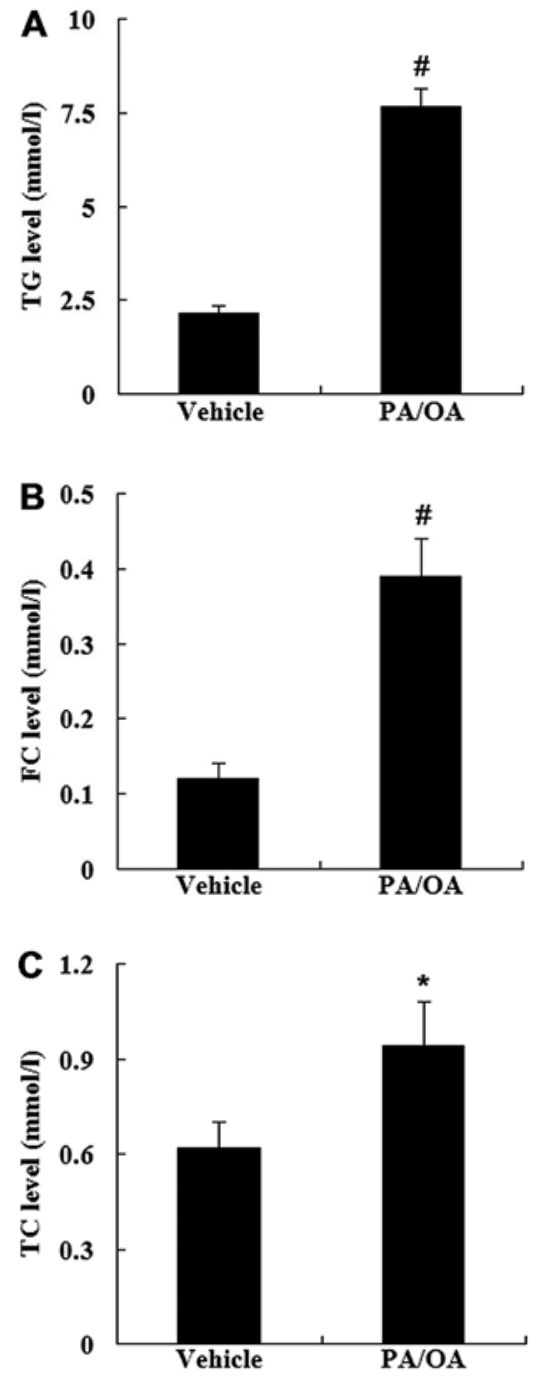

Figure 3. The levels of TG, FC and TC in the in vitro model of non-alcoholic fatty liver disease (NAFLD). (A) Levels of TG in the vehicle-treated and PA/OA-treated HepG2 cells. (B) Levels of FC in the vehicle-treated and PA/ OA-treated HepG2 cells. (C) Levels of TC in the vehicle-treated and PA/ OA-treated HepG2 cells. " $\mathrm{P}<0.05$ and ${ }^{\#} \mathrm{P}<0.01$ compared with the vehicle. TG, triglyceride; FC, free cholesterol; TC, total cholesterol; PA, palmitic acid; OA, oleic acid.

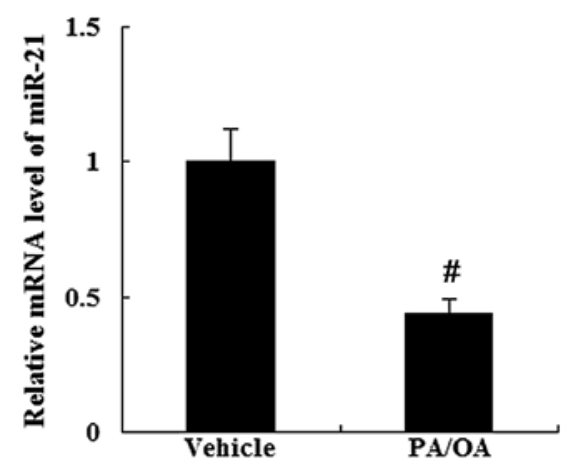

Figure 4. Expression of miR-21 in the in vitro model of non-alcoholic fatty liver disease (NAFLD). " $\mathrm{P}<0.01$ compared with the vehicle. PA, palmitic acid; OA, oleic acid.

As assessed by RT-qPCR and western blot analysis, the enhanced expression of miR-21 resulted in a significant 
A
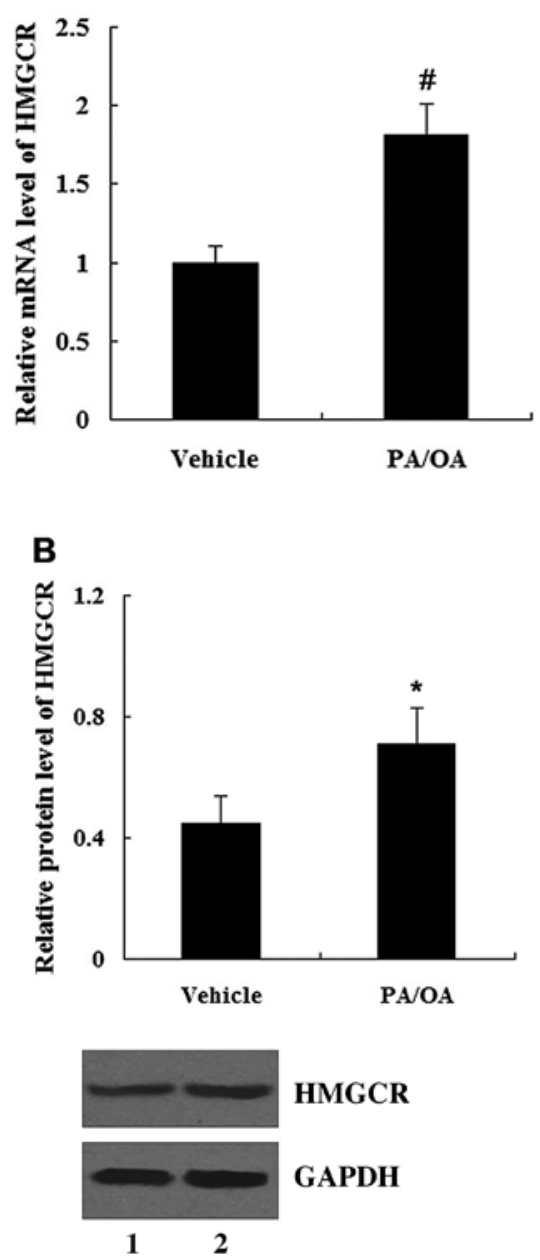

Figure 5. Expression of HMGCR in the in vitro model of non-alcoholic fatty liver disease (NAFLD). (A) Relative mRNA level of HMGCR. (B) Relative protein level of HMGCR. ${ }^{*} \mathrm{P}<0.05$ and ${ }^{\#} \mathrm{P}<0.01$ compared with the vehicle. Lane 1, vehicle group; lane 2, PA/OA group. HMGCR, 3-hydroxy-3-methylglutaryl-co-enzyme A reductase; PA, palmitic acid; OA, oleic acid.

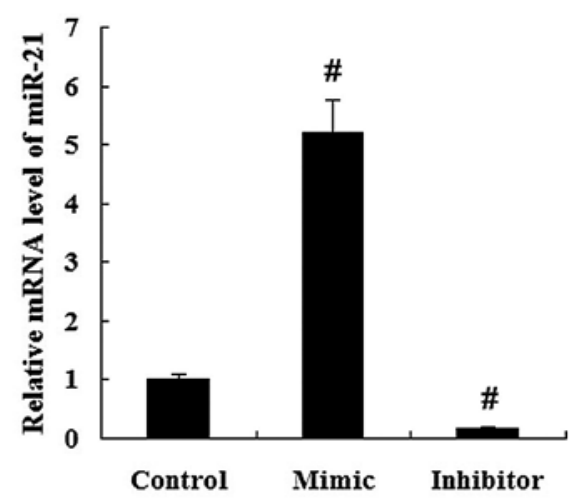

Figure 6. Expression of miR-21 in HepG2 cells transfected with the miR-21 mimic and miR-21 inhibitor. ${ }^{\#} \mathrm{P}<0.01$ compared with the controls.

decrease in HMGCR mRNA and protein expression. However, the suppression of miR-21 led to an increased HMGCR mRNA and protein expression (Fig. 8).

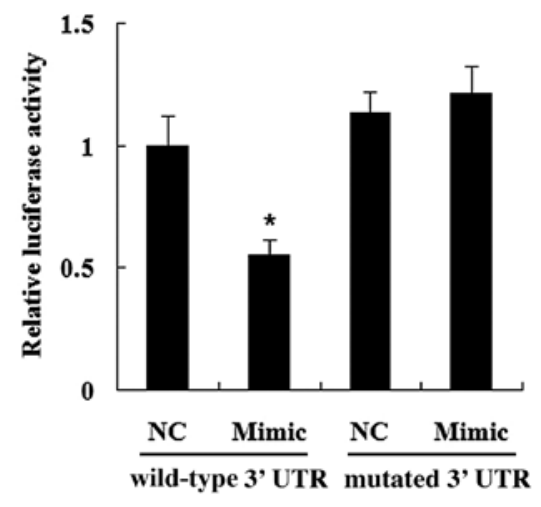

Figure 7. Relative luciferase activity of HepG2 cells transfected with the miR-NC/miR-21 mimic and HMGCR wild-type 3'-UTR/HMGCR mutated 3'-UTR. "P $<0.05$ compared with the NC. NC, negative control; UTR, untranslated region; HMGCR, 3-hydroxy-3-methylglutaryl-co-enzyme A reductase.
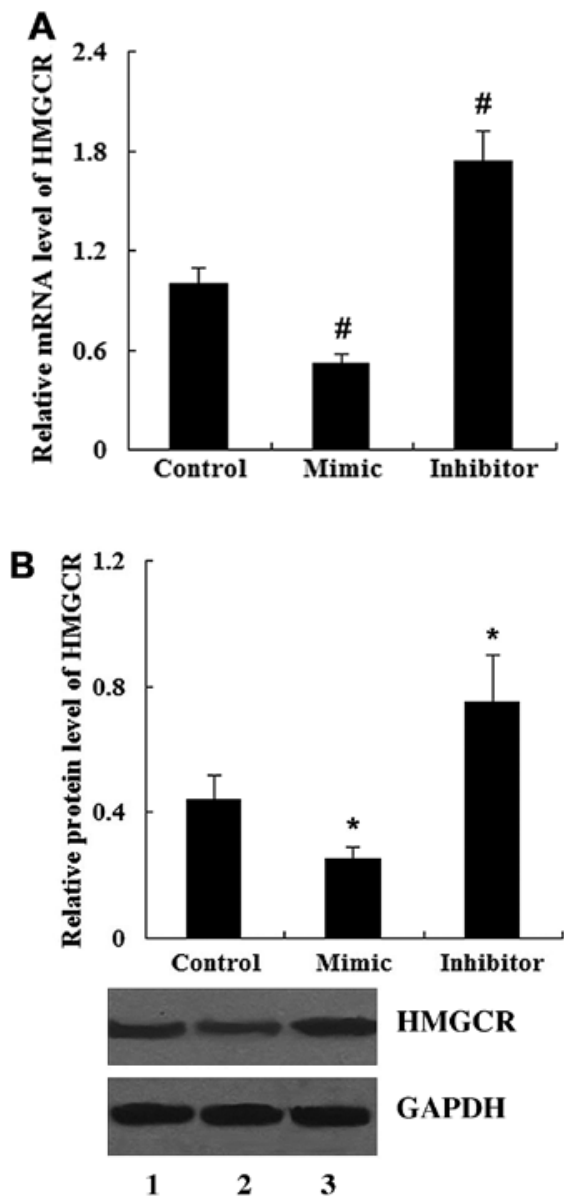

Figure 8. Expression of miR-21 in HepG2 cells transfected with the miR-21 mimic and miR-21 inhibitor. (A) Relative mRNA level of HMGCR. (B) Relative protein level of HMGCR. ${ }^{*} \mathrm{P}<0.05$ and ${ }^{~} \mathrm{P}<0.01$ compared with the controls. Lane 1, control group; lane 2, miR-21 mimic group; lane 3, miR-21 inhibitor group. HMGCR, 3-hydroxy-3-methylglutaryl-co-enzyme A reductase.

Effect of miR-21 on the levels of TG, FC and TC in the in vitro model of NAFLD. The miR-21 mimic was transfected into the vehicle-treated or PA/OA-treated HepG2 cells. As shown in Fig. 9, the expression of miR-21 was significantly increased in the miR-21-transfected cells compared with the miR-NC- 


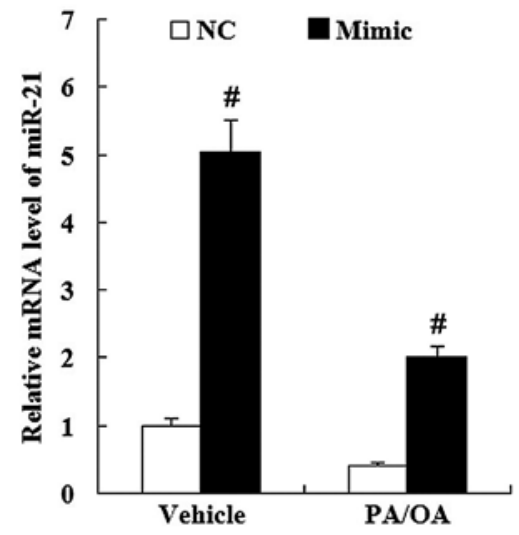

Figure 9. Expression of miR-21 in vehicle-treated and PA/OA-treated HepG2 cells following transfection with the miR-21 mimic. " $\mathrm{P}<0.01$ compared with the NC. NC, negative control; PA, palmitic acid; OA, oleic acid.
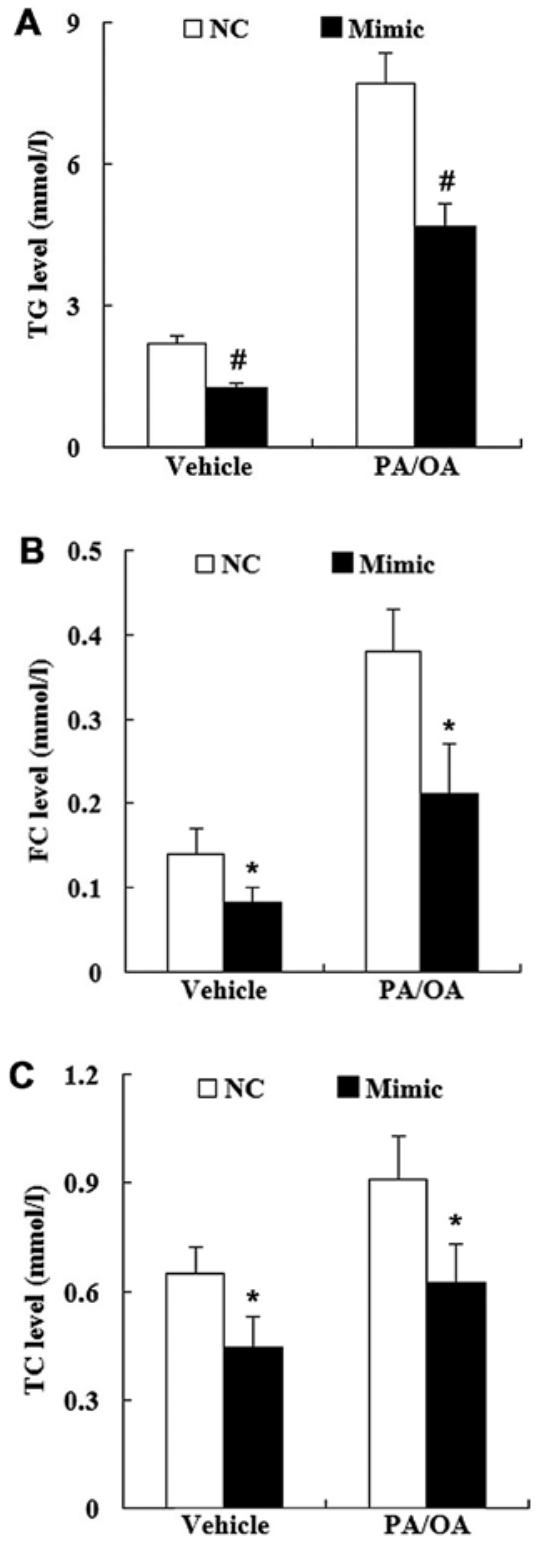

Figure 10. Levels of TG, FC and TC in the vehicle-treated and PA/OA-treated HepG2 cells following transfection with the miR-21 mimic. (A) TG level; (B) FC level; (C) TC level. " $\mathrm{P}<0.05$ and ${ }^{\#} \mathrm{P}<0.01$ compared with the NC. NC, negative control; TG, triglyceride; FC, free cholesterol; TC, total cholesterol; PA, palmitic acid; OA, oleic acid.
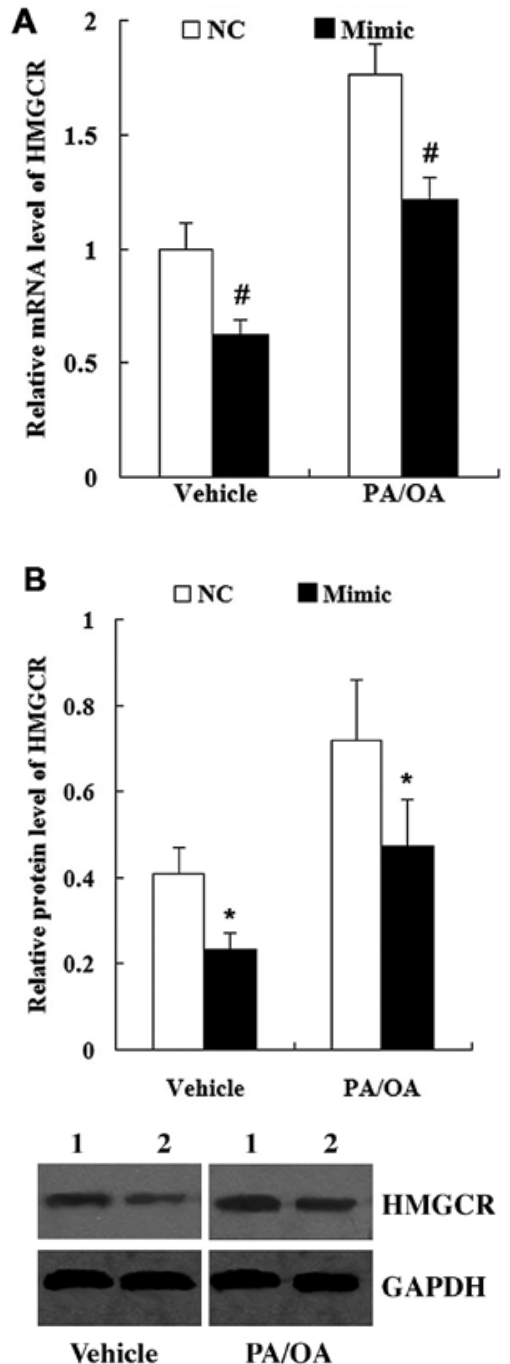

Figure 11. Expression of HMGCR in the vehicle-treated and PA/OA-treated HepG2 cells following transfection with the miR-21 mimic. (A) Relative mRNA level of HMGCR. (B) Relative protein level of HMGCR. " $\mathrm{P}<0.05$ and ${ }^{\text {}} \mathrm{P}<0.01$ compared with the NC. Lane $1, \mathrm{NC}$ group; lane 2, miR-21 mimic group. NC, negative control; PA, palmitic acid; OA, oleic acid. HMGCR, 3-hydroxy-3-methylglutaryl-co-enzyme A reductase.

transfected cells for both the vehicle-treated and PA/OA-treated HepG2 cells $(\mathrm{P}<0.01)$.

Using vehicle-treated HepG2 cells transfected with the miR-NC as the control, it was demonstrated that transfection with the miR-21 mimic significantly suppressed the level of TG in the vehicle-treated HepG2 cells. In addition, the levels of FC and TC were significantly decreased in the vehicle-treated HepG2 cells transfected with the miR-21 mimic. As for the PA/OA-treated HepG2 cells, the levels of TG, FC and TC were also decreased in the cells transfected with the miR-21 mimic compared with those transfected with miR-NC (Fig. 10).

Effect of miR-21 on HMGCR expression in the in vitro model of NAFLD. We then examined whether changes in miR-21 expression modulate HMGCR expression in PA/OA-treated HepG2 cells. As shown in Fig. 11, the enhanced expression of miR-21 not only downregulated HMGCR expression in the vehicle-treated HepG2 cells, but also in the PA/OA-treated HepG2 cells both at the mRNA and protein level (Fig. 11). 


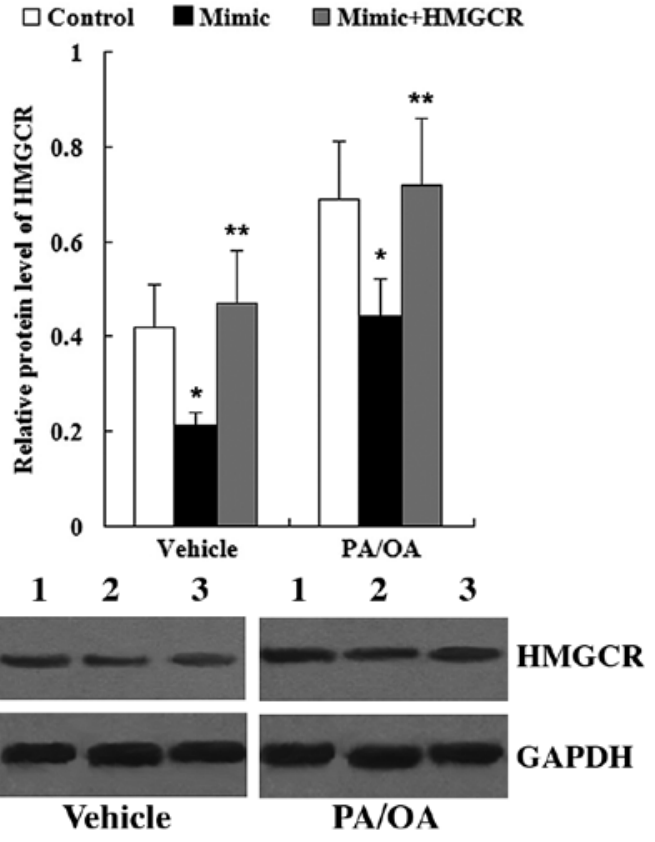

Figure 12. Protein expression of HMGCR in the vehicle-treated and PA/OAtreated HepG2 cells following transfection with the miR-21 mimic and HMGCR overexpression plasmid. " $\mathrm{P}<0.05$ compared with the control, ${ }^{* *} \mathrm{P}<0.05$ compared with the mimic. Lane 1, control group; lane 2, miR-21 mimic group; lane 3, miR-21 mimic + HMGCR overexpression plasmid group. HMGCR, 3-hydroxy-3- methylglutaryl-coenzyme A reductase; PA, palmitic acid; OA, oleic acid.

$H M G C R$ is involved in the effects of miR-21 on the levels of $T G, F C$ and $T C$ in the in vitro model of NAFLD. To determine whether HMGCR is involved in the effects of miR-21 on the levels of TG, FC and TC in PA/OA-treated HepG2 cells, the HMGCR overxpression plasmid was transfected into the HepG2 cells. As shown in Fig. 12, the HMGCR protein level was significantly increased in the cells transfected with the miR-21 mimic + HMGCR overexpression plasmid compared with the cells transfected with the miR-21 mimic; this was observed in both the vehicle-treated and PA/OA-treated HepG2 cells.

As shown in Fig. 13, compared with the cells transfected with the miR-21 mimic, following transfection with the HMGCR overexpression plasmid, the levels of TG, FC and TC were significantly increased in both the vehicle-treated and PA/OA-treated HepG2 cells.

\section{Discussion}

Ahn et al (17) found that the expression of miR-21 was decreased in the livers from mice fed a high-fat diet and in Hepa 1-6 cells treated with stearic acid (SA), and that miR-21 upregulation markedly blocked the SA-induced intracellular lipid accumulation. In this study, we investigated miR-21 expression in NAFLD both in vivo and in vitro. The results revealed that the serum level of miR-21 was lower in patients with NAFLD. Subsequently, in order to mimic the NAFLD condition in vitro, HepG2 cells were treated with PA and OA. The concentration of free fatty acids (FFA) used in the experiments was similar to the fasting FFA plasma concentrations observed in human $\mathrm{NASH}$, ranging from 700 to $1,000 \mu \mathrm{M}(18-21)$. This treatment
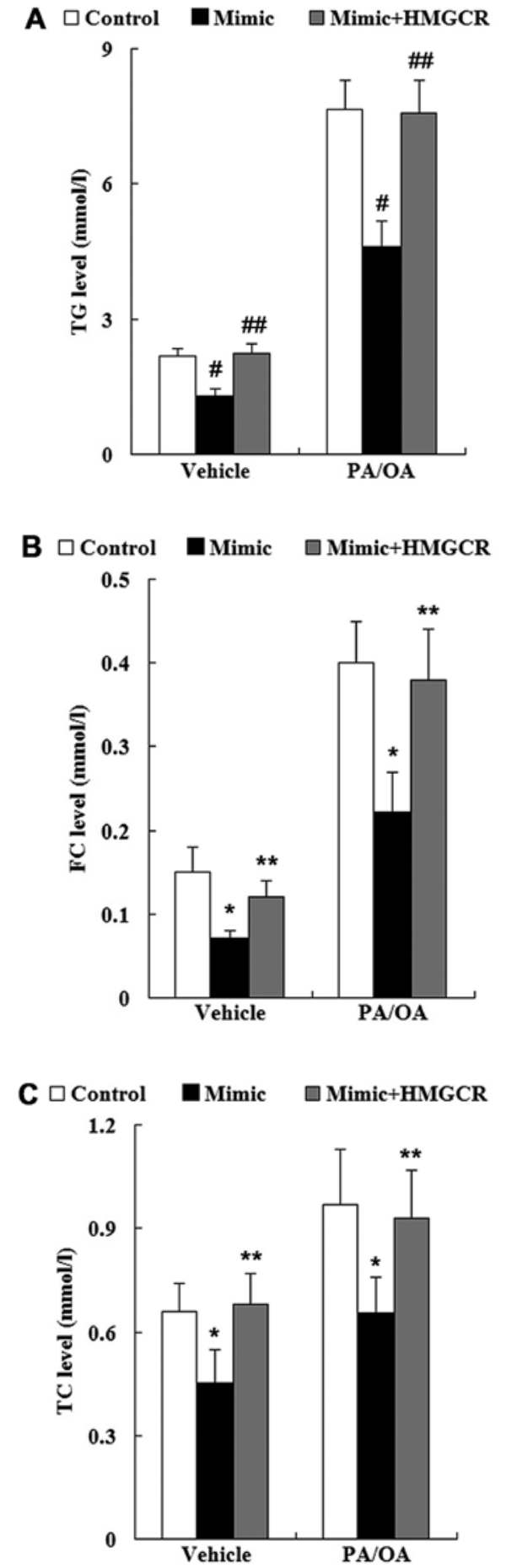

Figure 13. Levels of TG, FC and TC in the vehicle-treated and PA/OA-treated HepG2 cells following transfection with the miR-21 mimic and HMGCR overexpression plasmid. (A) TG level; (B) FC level; (C) TC level. "P<0.05 and ${ }^{\#} \mathrm{P}<0.01$ compared with the control, ${ }^{* *} \mathrm{P}<0.05$ and ${ }^{\# \#} \mathrm{P}<0.01$ compared with the mimic. HMGCR, 3-hydroxy-3-methylglutaryl-coenzyme A reductase; TG, triglyceride; FC, free cholesterol; TC, total cholesterol; PA, palmitic acid; OA, oleic acid.

mimics the FFA lipotoxicity and steatosis which occurs during the course of the disease (22). It was demonstrated that the levels of TG were significantly increased in the PA/OA-treated HepG2 cells; therefore, the in vitro model of NAFLD was successfully established. Consistent with the results from the in vivo experiments, the data revealed that miR-21 was also decreased in the in vitro model of NAFLD. 
A previous study found that HMGCR was relatively dephosphorylated in both phenotypes of NAFLD and thus in its active form $(23,24)$. Increased HMGCR expression is associated with NAFLD (25) and is related to FC levels and the severity of liver disease (26). In the present study, we examined the expression levels of HMGCR in patients with NAFLD and in an in vitro model of NAFLD. Both the in vivo and in vitro results demonstrated that HMGCR expression was upregulated in NAFLD.

Subsequently, we performed in vitro experiments to examine the effects of miR-21 on TG and cholesterol metabolism. It was demonstrated that miR-21 decreased the levels of TG, FC and TC in the PA/OA-treated HepG2 cells; this suggests that miR-21 regulates TG and cholesterol metabolism in NAFLD.

Cholesterol is mainly synthesized in the liver and cholesterol homeostasis is maintained by HMGCR transcriptional inhibition and enzyme modifications (27). HMGCR is the rate-limiting enzyme in the hepatic cholesterol biosynthesis pathway (28). HMGCR converts 3-hydroxy-3-methylglutarylcoenzyme A (HMG-CoA) to mevalonate and is the major target of cholesterol-lowering drugs (29).

Using prediction software (http://www.microrna.org/), HMGCR was identified to be a predicted target gene of miR-21. In the present study, to the best of our knowledge, we demonstrate for the first time that HMGCR is a direct target of miR-21 through luciferase reporter assay. In addition, RT-qPCR and western blot analysis revealed that HMGCR expression was altered following transfection with the miR-21 mimic or miR-21 inhibitor. These observations suggest an effect of miR-21 on both HMGCR transcript degradation and protein translation.

HMGCR overexpression plasmid was also transfected into vehicle-treated or PA/OA-treated HepG2 cells. It was found that HMGCR reversed the reducing effects of miR-21 on TG, FC and TC levels in NAFLD. Thus, these results suggest that the effects of miR-21 on TG and cholesterol metabolism in NAFLD are mediated through HMGCR.

Taken together, our data confirm that miR-21 is downregulated in the serum of patients with NAFLD. miR-21 regulated TG and cholesterol metabolism in our in vitro model of NAFLD, and this effect was achieved through the inhibition of HMGCR expression. The present study provides evidence that miR-21 may be a useful biomarker for the diagnosis and treatment of NAFLD.

\section{References}

1. Targher G, Bertolini L, Padovani R, et al: Prevalence of nonalcoholic fatty liver disease and its association with cardiovascular disease among type 2 diabetic patients. Diabetes Care 30: 1212-1218, 2007

2. Clark JM and Diehl AM: Hepatic steatosis and type 2 diabetes mellitus. Curr Diab Rep 2: 210-215, 2002.

3. Bedogni G, Miglioli L, Masutti F, Tiribelli C, Marchesini G and Bellentani S: Prevalence of and risk factors for nonalcoholic fatty liver disease: the Dionysos nutrition and liver study. Hepatology 42: 44-52, 2005.

4. Browning JD, Szczepaniak LS, Dobbins R, et al: Prevalence of hepatic steatosis in an urban population in the United States: impact of ethnicity. Hepatology 40: 1387-1395, 2004.

5. Vernon G, Baranova A and Younossi ZM: Systematic review: the epidemiology and natural history of non-alcoholic fatty liver disease and non-alcoholic steatohepatitis in adults. Aliment Pharmacol Ther 34: 274-285, 2011.
6. Fan JG: An introduction of strategies for the management of nonalcoholic fatty liver disease (NAFLD) recommended by Asia Pacific Working Party on NAFLD. Zhonghua Gan Zang Bing Za Zhi 15: 552-553, 2007 (In Chinese).

7. Ballestri S, Lonardo A, Bonapace S, Byrne CD, Loria P and Targher G: Risk of cardiovascular, cardiac and arrhythmic complications in patients with non-alcoholic fatty liver disease. World J Gastroenterol 20: 1724-1745, 2014.

8. Oni ET, Agatston AS, Blaha MJ, et al: A systematic review: burden and severity of subclinical cardiovascular disease among those with nonalcoholic fatty liver; should we care? Atherosclerosis 230: 258-267, 2013.

9. Nelson KM and Weiss GJ: MicroRNAs and cancer: past, present, and potential future. Mol Cancer Ther 7: 3655-3660, 2008.

10. Xu P, Guo M and Hay BA: MicroRNAs and the regulation of cell death. Trends Genet 20: 617-624, 2004.

11. Karp X and Ambros V: Encountering microRNAs in cell fate signaling. Science 310: 1288-1289, 2005.

12. Li S, Chen X, Zhang H, et al: Differential expression of microRNAs in mouse liver under aberrant energy metabolic status. J Lipid Res 50: 1756-1765, 2009.

13. Cheung O, Puri P, Eicken C, et al: Nonalcoholic steatohepatitis is associated with altered hepatic microRNA expression. Hepatology 48: 1810-1820, 2008.

14. Jin X, Ye YF, Chen SH, Yu CH, Liu J and Li YM: MicroRNA expression pattern in different stages of nonalcoholic fatty liver disease. Dig Liver Dis 41: 289-297, 2009.

15. Pogribny IP, Starlard-Davenport A, Tryndyak VP, Han T, Ross SA, Rusyn I and Beland FA: Difference in expression of hepatic microRNAs miR-29c, miR-34a, miR-155, and miR-200b is associated with strain-specific susceptibility to dietary nonalcoholic steatohepatitis in mice. Lab Invest 90: 1437-1346, 2010.

16. Cazanave SC, Mott JL, Elmi NA, et al: JNK1-dependent PUMA expression contributes to hepatocyte lipoapoptosis. J Biol Chem 284: 26591-26602, 2009.

17. Ahn J, Lee H, Jung CH and Ha T: Lycopene inhibits hepatic steatosis via microRNA-21-induced downregulation of fatty acid-binding protein 7 in mice fed a high-fat diet. Mol Nutr Food Res 56: 1665-1674, 2012.

18. Nehra V, Angulo P, Buchman AL and Lindor KD: Nutritional and metabolic considerations in the etiology of nonalcoholic steatohepatitis. Dig Dis Sci 46: 2347-2352, 2001.

19. Richieri GV and Kleinfeld AM: Unbound free fatty acid levels in human serum. J Lipid Res 36: 229-240, 1995.

20. Sanyal AJ, Campbell-Sargent C, Mirshahi F, et al: Nonalcoholic steatohepatitis: association of insulin resistance and mitochondrial abnormalities. Gastroenterology 120: 1183-1192, 2001.

21. Belfort R, Harrison SA, Brown K, et al: A placebo-controlled trial of pioglitazone in subjects with nonalcoholic steatohepatitis. N Engl J Med 355: 2297-2307, 2006.

22. Yao HR, Liu J, Plumeri D, et al: Lipotoxicity in HepG2 cells triggered by free fatty acids. Am J Transl Res 3: 284-291, 2011.

23. Beg ZH, Stonik JA and Brewer HB Jr: Phosphorylation of hepatic 3-hydroxy-3-methylglutaryl coenzyme A reductase and modulation of its enzymic activity by calcium-activated and phospholipid-dependent protein kinase. J Biol Chem 260: 1682-1687, 1985.

24. Beg ZH, Stonik JA and Brewer HB Jr: Modulation of the enzymic activity of 3-hydroxy-3-methylglutaryl coenzyme A reductase by multiple kinase systems involving reversible phosphorylation: a review. Metabolism 36: 900-917, 1987.

25. Caballero F, Fernández A, De Lacy AM, Fernández-Checa JC, Caballería J and García-Ruiz C: Enhanced free cholesterol, SREBP-2 and StAR expression in human NASH. J Hepatol 50: 789-796, 2009.

26. Min HK, Kapoor A, Fuchs M, et al: Increased hepatic synthesis and dysregulation of cholesterol metabolism is associated with the severity of nonalcoholic fatty liver disease. Cell Metab 15: 665-674, 2012.

27. DeBose-Boyd RA: Feedback regulation of cholesterol synthesis: sterolaccelerated ubiquitination and degradation of HMG CoA reductase. Cell Res 18: 609-621, 2008.

28. Trapani L, Segatto M, Simeoni V, et al: Short- and long-term regulation of 3-hydroxy 3-methylglutaryl coenzyme A reductase by a 4-methylcoumarin. Biochimie 93: 1165-1171, 2011.

29. Maron DJ, Fazio S and Linton MF: Current perspectives on statins. Circulation 101: 207-213, 2000. 